\title{
Machine Design: Redesigned
}

\section{Dr. George Youssef, California State University, Northridge}

Dr. George Youssef received his Ph.D. in Mechanical Engineering from University of California Los Angeles in 2010 and joined the faculty at California State University Northridge as Assistant Professor of Mechanical Engineering in 2011. His research interest is in the general area of solid mechanics with focus on nontraditional materials such as polymers, composites, and smart materials. His research contribution in dynamic properties of shock loaded materials, interfacial strength of direct bond wafers, environmental degradation of polymers, and biomechanics of walking. His research has been supported by National Science Foundation, Department of Defense, and private industries. Dr. Youssef was recognized by San Fernando Engineers Council as Distinguished Engineering Educator for 2014.

\section{Dr. J. Michael Kabo, California State University, Northridge}

Dr. Kabo received his PhD from UC Berkeley in Applied Mechanics in 1980. After a 23 year career conducting biomechanics research at UCLA he relocated to California State University, Northridge to become more engaged in teaching. He is currently a Professor in the Department of Mechanical Engineering where he also serves as Mechanical Engineering Graduate Coordinator and Course Coordinator for Machine Design. 


\title{
Machine Design: Redesigned
}

\begin{abstract}
Machine design is a cornerstone foundation course in any Mechanical Engineering program. The traditional objective of this course is to engage students with analysis techniques to guard against specific failure modes or to predict a product's life cycle based on a loading scenario. Generally, the course is taught by introduction of a topic first, e.g. static failure criteria, then examples are presented and homework sets are assigned to allow students to practice and sharpen their problem solving skills. The current methodology lacks the implementation of the complete engineering design process and the integration of other knowledge domains such as manufacturing. Additionally, the current course structure does not usually stimulate creativity necessary for the design process (ideation) or train students on decision making based on objective criteria. This paper presents an enhanced course structure developed over the past few years for a more comprehensive approach to machine design. The new course structure is hinged on the application of engineering design process, knowledge integration from prior courses as well as industrial practice, and adoption of design matrices as an objective decision making tool. We have retained many of the traditional pedagogies used in teaching machine design and supplement this educational experience with a significant project component based on current customer-need or economical challenge. Through the project students learn to: 1) create the design envelop based on a provided statement or requirement document; 2) define specific, meaningful, and measurable goals; 3) synthesize creative ideas to solve the problem; 4) perform a patent search to verify the innovative nature of their ideas; 5) produce a design matrix with evaluation criteria based on the goals and expected functionality; and 6) perform an in depth engineering analysis based on mechanics of materials, manufacturability, assembly, and packaging. The inclusion of an intensive writing and presentation experience with critical feedback engages students in a continuous reflection on the elements of the complete design process throughout the entire semester. It was found that this approach produces students who are better prepared for their senior design projects and engineering practice. Students noted an increase in their understanding of machine design concepts as an integration of all their prior preparatory training. The effectiveness of the revised course structure was evaluated through a survey of previous and current students.
\end{abstract}

\section{Introduction and background}

Prior to 2011, one of the common concerns of the Mechanical Engineering department's senior students was the inability to "engineer" or practice "design." This was also echoed by capstone project instructors. In other words, our students were not prepared to work on their senior design projects, unless they had prior industrial or volunteering experiences. Students were found to be unable to develop ideation and design skills independently prior to enrolling in their required culminating experience [1]. At our university, four different Mechanical Engineering capstone projects are available, all of which are competition-based. These projects include: Society of Automotive Engineers Formula Car Design and Competition, Intelligent Ground Vehicle Competition sponsored by IGVC, American Society of Mechanical Engineers Human Powered Vehicle Design and Competition, and AUVSI Unmanned Aerial Vehicle Competition. 
The inability of engineering graduates to engineer and design hampers the industrial productivity in the United States [1], since these students lack the ideation, design and practical skills that are in great demand by current employers. This issue is rooted in the lack of preparatory courses that inculcate the design process in our students early in their educational program. The traditional focus is on the important appropriate scientific and analytical techniques with little regard to the complexity of the design process and its applications. Traditionally, machine design courses, where design in some instances is first introduced, are focused on the analysis of stresses due to applied loads, static failure theories for ductile and brittle materials, fatigue, and analysis of mechanical components, such as shafts, fasteners, gears, etc. [2]. Notably, mechanical design textbooks are full of practical knowledge but it is presented solely from the perspective of performing a careful detailed analysis. Academic professors without considerable industrial experience promotes the basics of problem solving, in other words the 'science of analysis' [2]. Those academicians with extensive industrial experience understand the critical role design plays in the understanding of mechanical design and are able to share this experience with their students but may not possess the breadth of experience to cover all of the content equally in a full term course. As a result a large gap exists between the senior design experience and industry expectation and the knowledge attained in a typical machine design course.

The design process, sometimes referred to as the design cycle, exposes engineering students to two important lessons: 1) the design is iterative process [3]; and 2) design is a process of tradeoffs. In a typical design exercise, more than one solution exists and an engineer is expected to objectively evaluate all suitable solutions and decide on which to move forward with, i.e., tradeoff. Thereafter, the selected conceptual design is modeled and analyzed in detail. The design cycle thus consists of two symbiotic phases: synthesis and analysis. Furthermore, the design will continuously evolve through many iterations until the final design meets all the established design criteria. If an engineering student approaches a project beginning with the foundation of the design process, involving iteration and trade-off through synthesis and analysis, the result is an engineer who is ready to contribute to the workforce on the first day on the job.

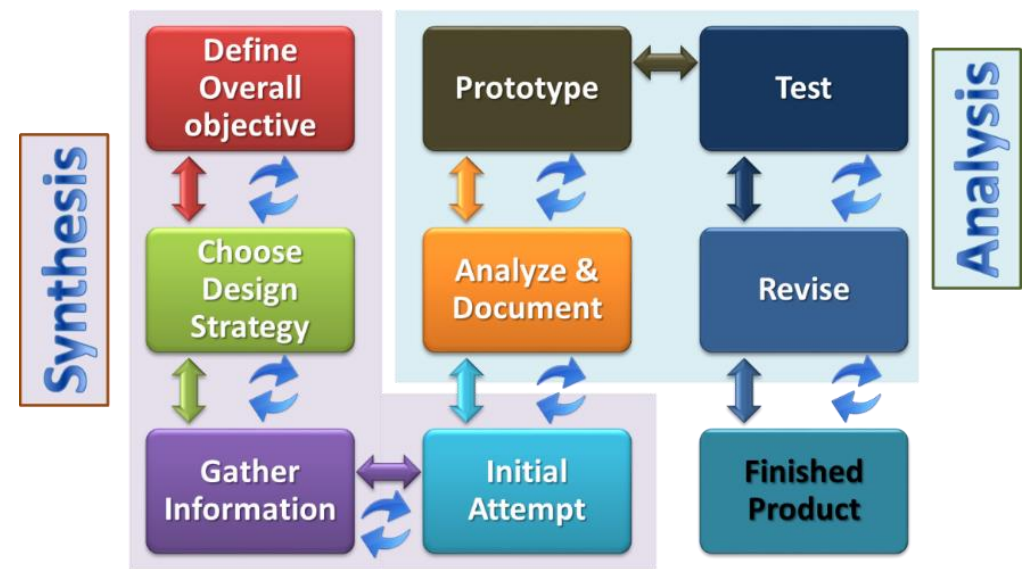

Figure 1: Engineering Design Cycle (modified from [3]).

Figure 1 depicts one model of the steps of the design process [3]. The design project topic is introduced at the earliest opportunity at the start of the term. The project topic may range from relatively simple to extremely complex in its subtleties. The intent is to stimulate ideation and 
finding solutions to issues that arise every step of the way in teamwork setting. Each group consists of 3-5 students. The project provides a term long framework whereby all of the elements of mechanical design can be introduced with contextual relevance. It begins with the definition of the overall objective of the project. In this step, the design requirement document is carefully reviewed and questions or concerns are articulated and communicated to the customer (the instructor). In our experience, most students never contemplate additional possibilities or alternatives when a design task is assigned. This is of high importance to the consumer or contractor who is expecting to be involved in the decision making process even though they might not completely comprehend the complexities of a proposed design. In the requirement document, the overall customer need, problem or challenge is specified as well as the machine functionality, design constraints, rules and regulations, and allocated resources such as budget and timeline. Once the requirement document is well-understood, the design team needs to define the overall design goal. It is encouraged that specific, meaningful, and measureable (SMM) goals to be defined. Here, in an academic setting, the professor can assist the students in thinking, focusing on and defining SMM goals in design courses. The second step is choosing an appropriate design strategy, in which the decision must be made whether the product is going to be mechanical, electrical, software, or hybrid, such as an automated machine or process. The design approach must be decided as well, whether top-down or bottom up. Additionally, decisions must be made regarding manufacturing processes versus the use of commercially available components. The third step is collecting information and conducting research about the design strategy and all components and subsystems. Moreover, in this step, research needs to be conducted about theories, techniques, and scientific principles to facilitate the subsequent steps in the design. We argue that devoting appropriate time and effort in the first three steps of the process will have a significant impact on the design by shortening the overall project time and reducing the number of iterations required. The result will be a more efficient process leading to the final product design that meets all the required design criteria. Put another way, the first three steps in design process should be allocated enough resources, in effect time, funds, effort, expertise, to achieve "lean design," and making informed decisions. Only then, initial design attempts are done. If the preceding steps are executed correctly, the initial attempts will satisfy the design requirements with implied novelty, involving creative and innovative solutions. In the initial attempt and based on the research, the designer is now considering the manufacturing, assembly, and the packaging as well as the loading scenarios. These first four steps constitute the synthesis phase of the design cycle. Subsequently, the selected solution can be analyzed based on the loading scenarios and environmental conditions to define the shape, size, and materials. The mechanical analysis can be performed either using closed-form analytical methods as usually discussed in machine design courses or numerical methods that are discussed in finite element analysis courses. Concurrently, a clear, concise and inclusive documentation of the design analysis should be compiled.

Historically, engineers are notorious for leaving documentation to the eleventh hour and not being inclusive in reports. To counter this deficiency, students are required to keep a design log that is collected and reviewed periodically. Once the analysis step is concluded, a prototype is built from represented materials at 1:1 scale, which is then tested to validate the functionality of the product. If testing is successful, then the product is moved to production. Otherwise, revision will be required to meet the design requirements. However, revision is included as one step of the design cycle, it should be noted that iterating is implied also between subsequent steps 
(marked as revolving arrows in Figure 1). Due to the intensive nature of requiring students to focus largely on the synthesis and analysis aspects of the design process, there is usually insufficient time left in a given term for students to actually reach the prototype stage of the design cycle.

In a typical mechanical engineering curriculum students become so enthralled and enamored with the wonderful tools available at their disposal that they lose sight of the ability to devote adequate time to generate a design concept that actually has merit. This, in turn, results in graduating mechanical engineers that are very attached to 3D modeling software but do not have sufficient knowledge to create executable mechanical designs that are truly creative and innovative. In the remaining section, successful approach to overcome this problem is presented, in which the design cycle is integrated into a real-life design project in a machine design course. Results from the reported approach are then presented.

\section{Approach}

This paper reports on a project-based approach to teach machine design, in which a balance is struck between traditional analytical and scientific techniques, which includes a focus on creativity and ideation, to develop various machine components based on mechanics of materials approach, and the practice of design. The assigned projects are based on contemporary issues, which helps to intrigue and engage the students. The project ideas ranged from an apparatus to recycle cans and bottles, to under-the-desk exercise machines, to PC hard-disk destruction equipment, and to manufacturing process automation. The project is assigned over one semester with milestones that are drawn from the design cycle presented above. Indeed, project-based learning is not new in engineering education [4-5], but the uniqueness of our approach is the integration of the design cycle into a project that requires in-depth analysis using hand calculations. Therefore, the students gain the required scientific knowledge to perform analysis as well as the practical experience of real-life design. Significant emphasis is given to documentation and report writing. Figure 2 shows the details of adopted approach, which was implemented in our junior level machine design course.

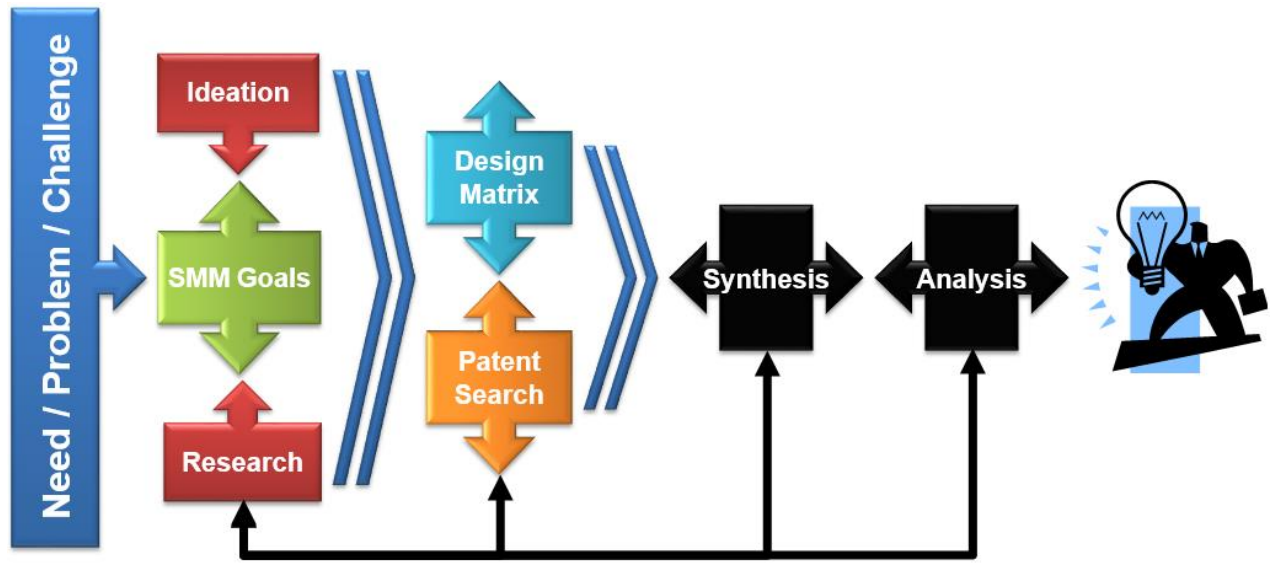

Figure 2: Integration of design cycle in machine design course. 
At the beginning of the semester (15 weeks), students are divided into groups that consist of 3-4 people at most and are presented with the project statement. A self-grouping method where students recruit their teammates or random assignment to a group is used to form the teams. The project statement is considered as the design requirement document. A design factor of safety (FoS) of 4 is imposed on all design components (as appropriate) for all teams to allow them to: 1) practice iterative design; and 2) understand the effect of FoS on synthesis and analysis. Initially the students are required to submit a summary of their initial design idea for approval by the instructor. The project idea is approved or disapproved with comments as appropriate. In some cases the team may be encouraged to completely redirect their focus because in the instructor's opinion the members of the team do not have sufficient experience between them to accomplish their proposed work. Examples would be projects that would require electromechanical control systems when none of the students have completed systems dynamics or introduction to controls courses or the anticipated use of a four-bar linkage when none of the team members has had any formal training in kinematics. In such cases, the students are advised to develop an alternative idea.

The students spend approximately one week understanding the requirements and defining their perspective team goals. Students are required to develop 3-5 different design alternatives. Thereafter, they start gathering information and conduct research about the different design approaches they develop. Students then submit an "ideation report," in which they include handsketches and narrative of the considered solutions. Based on the feedback provided by the professor on the ideation report, students create a design evaluation matrix by defining specific criteria that are driven from the team goals and project requirements. Using the design matrix, students objectively evaluate each of the proposed solutions and decide on the solution that would move forward for further consideration. Simultaneously, with the design matrix, students perform patent search using the United States Patent and Trade Mark Office (www.uspto.gov) and Google (www.patent.google.com) patent databases. In their patent search, students use keywords extracted from the ideation report. Thereafter, each group compiles and submits the second report, which is the "Patent Search and Design Matrix" report.

Once the second report is graded, effectively approving their design selection, students begin material selection, assigning dimensions, analysis and solid modeling of the selected design and prepare for Preliminary Design Review (PDR). PDR is done in two different ways, depending on the professor in charge, which are focus-group style and in-class presentation. In the focus-group approach, the instructor and each team meet to discuss the details of the proposed design with emphasis on the design logic, material candidates, manufacturing processes, assembly, and packaging. In the in-class approach, students present the details of their designs to their classmates and the professor. Students are exposed to different approaches by their peers and are encouraged to ask questions of a critical nature. Regardless of the PDR method, students are challenged to think about every aspect of their design as in professional industrial settings. This phase is usually done during the seventh or the eighth week of the semester.

After PDR, students are encouraged to consider the modifications discussed during the preliminary review and make necessary changes. Teams then focus on performing design analysis and calculations based on the machine design knowledge discussed in the lectures. The specific analyses used are selected based on their appropriateness for the project. Students 
perform analysis based on static failure theories, where they decide on the appropriate failure criteria based on their material's selection and confidence in defining the boundary conditions. They also perform fatigue analysis on components that undergo repeated dynamic loadings. For those projects that require a motor the students must identify torque, horsepower, and RPM needs derived from their analysis. Upon the conclusion of the analysis, students present their findings in Critical Design Review (CDR) presentation, to simulate workplace environment, write the final report, and create complete product definition (engineering drawings and models).

It is worth noting that there are many skills integrated in our approach, which are aligned with National Academy of Engineering report on Engineers of 2020 [6] and Accreditation Board for Engineering and Technology (ABET) (www.abet.org) students learning outcomes (outcomes b and c). Students gain and sharpen skills such as: understanding requirement documents, defining goals, synthesis, design evaluation, making decision, teamwork, written communication, oral communication, conduct patent search, creativity, innovation, perform in-depth mechanical analysis, problem solving, and machine design. Since the implementation of this approach, capstone project instructors have noticed significant improvement in the ability of students synthesize and analyze, and most importantly, the practice of design.

\section{Results and discussion}

To evaluate the effectiveness of the aforementioned pedagogical approach, a self-perception anonymous short survey that consisted of 13 questions was administrated online to previous students enrolled in this redesigned course. In the first two questions, participants were asked to indicate their academic status (graduated or still student), and in which industry they are currently employed. Two other questions inquired about the impact of the new approach in student's preparedness for the capstone design project and subsequent design courses. The remaining questions asked the students to evaluate the impact of each of the project activities on preparing them. A total of 224 students, who were enrolled in the machine design course between Fall 2012 and Spring 2014, were asked to voluntarily participate in the survey. 76 responses were received $(\sim 34 \%)$, of which 66 responded within five days of posting the survey.

Figure 3a shows the percentage of participants who are still students (53\% of responses) and those who already graduated ( $47 \%$ of responses). Figure $3 \mathrm{~b}$ illustrates that the participants are distributed in wide-range of industries from aerospace to research and development. That is, the skills attained from the new course redesign can be assessed from academic and industrial perspectives. 

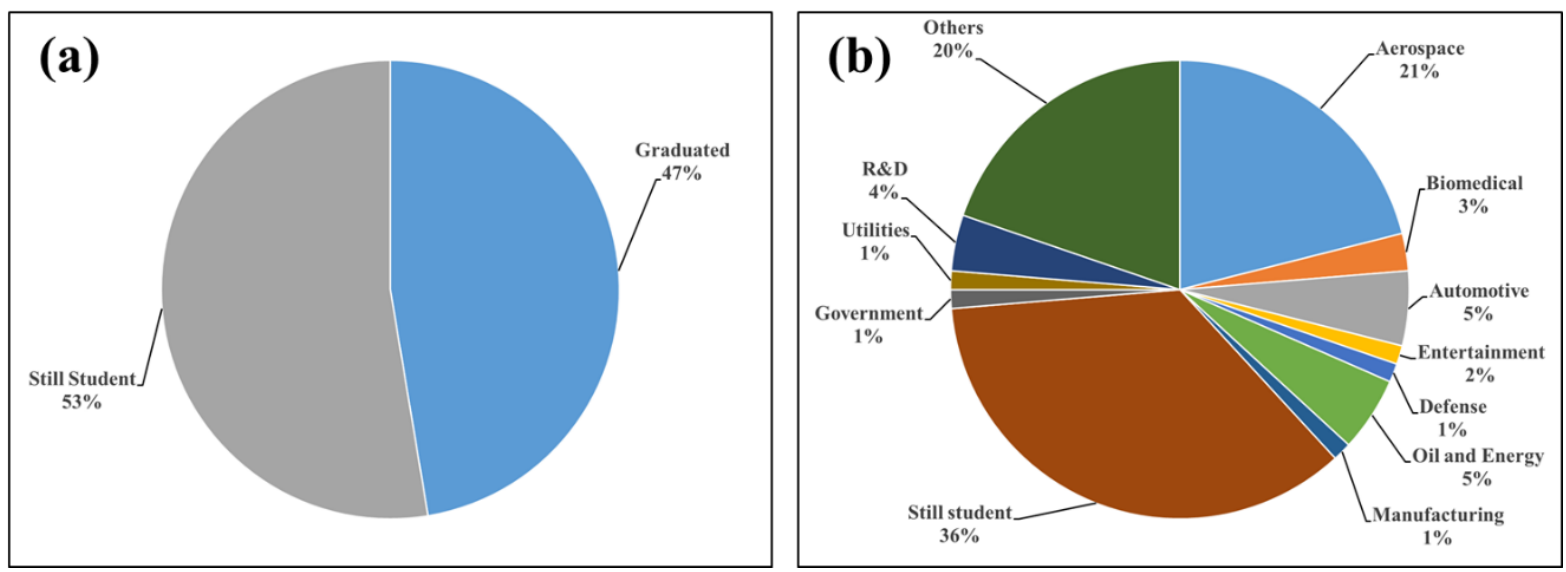

Figure 3: Percentage of (a) student and graduates; and (b) industry sector.

Table 1 shows the survey questions, where each participant were asked to rate (on the scale 1 to 5 , where 1 represented "not well" and 5 indicated "extremely well") the level the machine design course prepared them. It also displays the survey results, where it details the percentage of students rated the course impact as above average including the mean, median, mode, and variance statistics. Notably, the mode of all the questions in the survey is 5, which is the highest grade and depicts that the majority of the participants indicated the new approach has impacted them extremely well. In general, participants had a very favorable opinion, median $\geq 4$, of the impact of the integration of fundamental knowledge of machine design with the design cycle. Nonetheless, the participants indicated that despite the course introducing them to patent and reference literature searches, it requires some improvement and refinement since only $57 \%$ of responses rated the patent search activity as having "above average" utility.

\section{Conclusion}

This paper presented a unique approach to teach machine design course, in which we coupled the classical method of problem solving using analytical and scientific methods with a project that is based on contemporary issues. The conduct of the course and the demands placed on the students and the instructor are intense. The uniqueness of the presented approach is in the integration of engineering design cycle with project-based learning and classical pedagogies of machine design. This approach places significant additional demands on the instructor to focus on comprehensive critical review of submitted documents several times during an individual term. This approach infused desirable skills in our students and prepared them to be effective members of design teams in academia and industry.

\section{Bibliography}

[1] Liebman, Jon C. "Designing the design engineer." Journal of professional issues in engineering 115.3 (1989): 261-270. 
[2] Tian, Fang. "Improve Teaching Quality of Machine Design Course by Comprehensive Application of Multiple Methods." 2014 International Conference on Education Reform and Modern Management (ERMM-14). Atlantis Press, 2014.

[3] Horenstein, Mark N. Design Concepts for Engineers (ESource Series). Prentice-Hall, Inc., 2005.

[4] Dutson, Alan J., et al. "A Review of Literature on Teaching Engineering Design Through Project-Oriented Capstone Courses." Journal of Engineering Education 86.1 (1997): 17-28.

[5] Dym, Clive L., et al. "Engineering design thinking, teaching, and learning." Journal of Engineering Education 94.1 (2005): 103-120.

[6] Clough, G. Wayne. "The engineer of 2020: Visions of engineering in the new century." National Academy of Engineering, Washington, DC (2004). 
Table 1: Results from student survey (No. of responses $=76$ ).

\begin{tabular}{|c|c|c|c|c|c|}
\hline Question - Rate the level ME330 prepared you for: & $\begin{array}{l}\text { Above } \\
\text { Avg. }\end{array}$ & Mean & Median & Mode & Variance \\
\hline Senior design course. & $72 \%$ & 4.0 & 4.0 & 5.0 & 1.1 \\
\hline Subsequent design courses. & $76 \%$ & 4.1 & 4.0 & 5.0 & 0.9 \\
\hline Read and understand Design Requirement Documents. & $72 \%$ & 3.9 & 4.0 & 5.0 & 1.5 \\
\hline Define Design Goals. & $78 \%$ & 4.2 & 4.0 & 5.0 & 0.8 \\
\hline Synthesize creative and innovative designs. & $70 \%$ & 3.9 & 4.0 & 5.0 & 1.3 \\
\hline Perform Patent Search. & $57 \%$ & 3.7 & 4.0 & 5.0 & 1.3 \\
\hline $\begin{array}{l}\text { Effectively document, report, and communicate design activities } \\
\text { and findings. }\end{array}$ & $78 \%$ & 4.1 & 4.0 & 5.0 & 0.9 \\
\hline Participate in Preliminary Design Review. & $78 \%$ & 4.2 & 4.0 & 5.0 & 0.9 \\
\hline Participate in Critical Design Review. & $79 \%$ & 4.3 & 5.0 & 5.0 & 0.9 \\
\hline Objectively evaluate designs using Design Matrix. & $78 \%$ & 4.1 & 4.0 & 5.0 & 1.0 \\
\hline $\begin{array}{l}\text { Perform in-depth engineering analysis based on mechanics of } \\
\text { materials, manufacturability, assembly, and packaging. }\end{array}$ & $72 \%$ & 3.9 & 4.0 & 5.0 & 1.6 \\
\hline
\end{tabular}

